\title{
A cardiovascular collapse following vigorous cough during spinal anesthesia
}

\author{
Hyun Jee Kim and Ji Seob Kim \\ Department of Anesthesiology and Pain Medicine, Keimyung University School of Medicine, Daegu, Korea
}

Violent coughing can trigger symptomatic hypotension and may further lead to a complete loss of consciousness, which is considered a 'cough syncope' in the latter case [1]. We experienced a case of cardiovascular collapse after coughing under spinal anesthesia. We report on the case with a review of the relevant literatures.

A 73-year old man was scheduled to undergo an emergency cystoscopic evacuation and transurethral resection of the prostate (TURP) under spinal anesthesia, due to gross hematuria. He weighed $55 \mathrm{~kg}$ and was $160 \mathrm{~cm}$ tall. He had a history of hypertension lasting for 5 years and received treatment with diuretics. $\mathrm{He}$ arrived at the operating room without premedication. His vital signs were a 120/75 $\mathrm{mmHg}$ of blood pressure (BP), heart rate (HR) of 75-78 beats/min with normal sinus rhythm and $99 \%$ of oxygen saturation $\left(\mathrm{SpO}_{2}\right)$. With the patient in the sitting position, a 25-gauge Whitacre spinal needle was inserted at the third to fourth lumbar spinal interspace; and $6 \mathrm{mg}$ of $0.5 \%$ hyperbaric bupivacaine, mixed with $15 \mu \mathrm{g}$ of fentanyl, was injected. Sensory block to cold with an alcohol swab was tested bilaterally 5 and 10 minutes after the injection; they were T12 and T10, respectively. At the start of operation, 10 minutes after the subarachnoid block, his vital signs were a 95/60 mmHg of BP, 65-70 beats/min of $\mathrm{HR}$ and $99 \%$ of $\mathrm{SpO}_{2}$. During the surgery, sedative drug was not administered, and the patient had intermittent conversations with the anesthesiologist. He did not complain of dizziness, nausea or other discomforts. Fifteen minutes after the start of surgery, 25 minutes after the subarachnoid block, the patient had a very loud cough, once. Immediately after the cough, HR decreased abruptly from 65 beats/min to 30 beats/min, with sinus rhythm. Simultaneously the patient lost consciousness and did not breathe at all, and noninvasive blood pressure could not be estimated. An intravenous atropine $1 \mathrm{mg}$ was administered with manual mask ventilation, with $100 \% \mathrm{O}_{2}$. His heart rate increased to 100 beats/min, but BP was 55/30 mmHg. A tracheal intubation was performed, and mechanical ventilation was applied with $100 \% \mathrm{O}_{2}$. Epinephrine $0.1 \mathrm{mg}$ was administered intravenously with rapid hydration and Trendelenburg position. Invasive arterial BP monitoring through the right radial artery was performed (BP was 60-65/30-32 $\mathrm{mmHg}$; and heart rate increased to 110 beats/min). We inserted central venous catheter in the right internal jugular vein (central venous pressure: 6 $\mathrm{mmHg}$ ). The transesophageal echocardiography (TEE) demonstrated no evidence of LV systolic dysfunction or RV wall motion abnormality or thrombus at any angle. Hypovolemia was diagnosed; and continuous volume therapy and packed RBSs were administered. Phenylephrine infusion was maintained. Following vital sign stabilization, the surgery proceeded. During the surgery, volume status was evaluated through TEE and central venous pressure. Three units of packed RBCs and $1000 \mathrm{ml}$ of cystralloid were administered. Postoperative brain MRI findings were unremarkable, and the patient recovered consciousness 25 hours after arriving at the intensive care unit and was extubated at postoperative day (POD) 2. The patient was discharged at POD 6 without any complications.

Although being widely known to clinicians, the pathophysiology of cough syncope has not been completely understood owing to its uncommon occurrence. Sustained coughing can elicit decreased venous return and reduced cardiac output, fol-

Corresponding author: Hyun Jee Kim, M.D., Ph.D., Department of Anesthesiology and Pain Medicine, Keimyung University School of Medicine, 56, Dalseong-ro, Jung-gu, Daegu 700-712, Korea. Tel: 82-53-250-7232, Fax: 82-53-250-7240, E-mail: hyunjee@kmu.ac.kr (c) This is an open-access article distributed under the terms of the Creative Commons Attribution Non-Commercial License (http:// creativecommons.org/licenses/by-nc/3.0/), which permits unrestricted non-commercial use, distribution, and reproduction in any medium, provided the original work is properly cited. 
lowing increased intrathoracic pressure [2]. Recently, in intermittent coughs, an increased forearm blood flow was observed in patients, and baroreflex mechanism was strongly supported [3]. Cough syncope is generally classified as a reflex syncope [1]. Reflex syncope refers to conditions in which cardiovascular reflexes turn up in an inappropriate way, which are originally useful in maintaining the circulation. Although cough syncope belongs to the neutrally-mediated reflex syncope along with vasovagal syncope, it has been reported that the origins of cough syncope triggers are distinct from those of vasovagal syncope, given the different results of head-up tilt test [3]. Cough syncope shares clinical characteristics with vasovagal syncope. Cough syncope is due to abnormally increased vagal tone and decreased sympathetic tone, which lead to relative or absolute bradycardia, hypotension and cerebral hypoperfusion.

In the present case, the spinal blockade level was proper for both performing the surgery and maintaining the patient's circulation. The patient was kept NPO for 12 hours owing to the prolonged work-up time and had a hematoma in the bladder. This pre-existing dehydrated status was considered when the initiation of anesthesia and pre-hydration before the injection were performed. But during the surgery, ongoing bleeding from the surgical site may have aggravated his hypovolemia. The irrigation fluid used in this surgery was normal saline, with the use of bipolar resectoscope. Arterial blood gas analysis before the TEE examination showed a $\mathrm{pH}$ 7.32, $\mathrm{PaCO}_{2} 42 \mathrm{mmHg}, \mathrm{PaO}_{2}$
$155 \mathrm{mmHg}, \mathrm{Na}^{+} 139 \mathrm{mEq} / \mathrm{L}, \mathrm{K}^{+} 3.1 \mathrm{mEq} / \mathrm{L}$ and hematocrit $32 \%$. These imply the low possibility of the relevance of this case with the TURP syndrome. The postoperative serum osmolality was in a normal range.

Typical cough syncope which is classified as reflex syncope is a situational syncope and has no specific underlying disease. This patient did not have any underlying disease and showed no abnormalities in carotid sinus massage and head-up tilt test. Moreover, syncope after coughing was not reproduced after the surgery in this patient. Therefore, this case does not fit the typical cough syncope, and it cannot be concluded that this event is originated exclusively from cough syncope. We suggest that this case could be caused by excessive vagal activation, which could not be compensated in the context of ongoing hypovolemia and limited cardiopulmonary reserve in this elderly patient with hypertension. While previous reported cases of vasovagal circulatory collapse had discussed the patients' anxiety during spinal anesthesia [4], this patient was calm and relaxed without the sedative drugs.

In conclusion, exact estimation of volume status and keeping of normovolemia are important especially to provide for an abrupt adverse event, such as a sudden increase in baroreflex activity, during spinal anesthesia. Moreover, a careful prediction of intraoperative surgical complications that affect the patient's vital signs can lead to a safer choice of anesthesia method.

\section{References}

1. Saklani P, Krahn A, Klein G. Syncope. Circulation 2013; 127: 1330-9.

2. Sharpey-Schafer EP. The mechanism of syncope after coughing. Br Med J 1953; 2: 860-3.

3. Benditt DG, Samniah N, Pham S, Sakaguchi S, Lu F, Lurie KG, et al. Effect of cough on heart rate and blood pressure in patients with "cough syncope". Heart Rhythm 2005; 2: 807-13.

4. Jang YE, Do SH, Song IA. Vasovagal cardiac arrest during spinal anesthesia for Cesarean section -A case report. Korean J Anesthesiol 2013; 64: $77-81$. 